Case Report

\title{
Mirror Image Subarachnoid Haemorrhage: An Unusual Presentation of Internal Carotid Artery Aneurysm
}

\author{
Vikas Kumar, Vinayak Rastogi, Daljit Singh*, Hukum Singh
}

Department of Neurosurgery, G. B. Pant Institute of Postgraduate Medical Education \& Research, New Delhi, India.

\section{*Corresponding author: Singh, D. Department of Neurosurgery,G. B. Pant Institute of Postgraduate Medical Education \& Research, New Delhi, India. Tel: +91 9718599353; E-mail: drdaljit@hotmail.com}

Citation: Singh, D., et al. Mirror Image Subarachnoid Haemorrhage: An Unusual Presentation of Internal Carotid Artery Aneurysm(2015) Int J Neurol Brain Disord 2(1): 23- 24.

\section{Received Date: May 17, 2015 \\ Accepted Date: June 18, 2015 \\ Published Date: June 22, 2015}

Keywords: Aneurysmal subarachnoid haemorrhage; Mirror subarachnoid haemorrhage; Mirror aneurysm; Intracranial aneurysm

\begin{abstract}
We report a case of a patient who was referred to us for the treatment of right internal carotid supraclionoid aneurysm with subarachnoid haemorrhage on right side. In the evening before surgery, the patient had severe headache and loss of consciousness. Computed tomography scan of head revealed subarachnoid haemorrhage on the left side. A suspicion of a mirror aneurysm arose. Patient underwent digital subtraction angiography which showed same right internal carotid artery supraclionoid aneurysm which had increased in size. This is the first description of mirror subarachnoid haemorrhage which happened with a single aneurysm and is different from mirror aneurysm. The aneurysm was treated with endovascular coiling. A review of pathophysiology, radiological imaging, clinical manifestations and management is presented and discussed.
\end{abstract}

\section{Introduction}

Mirror aneurysms are paired aneurysms found within similar distributions on bilateral intracranial arteries. Their incidence ranges from less than $1 \%$ to $9.4 \%$ and are more common in patients with multiple intracranial aneurysms ${ }^{[1,2]}$. They may present with subarachnoid haemorrhage (SAH) on both sides of brain which looks like mirror image of each other. These are grossly picked on as incidental twin aneurysm on contralateral side. The most common clinical presentation of mirror aneurysm is subarachnoid haemorrhage. Mirror image SAH following re-rupture of a single intracranial aneurysm is not reported. We present a case of a single aneurysm that presented with a mirror image SAH on two different occasions.

\section{Case Summary}

A 45 years old female patient, who had acute onset of severe headache with vomiting was admitted elsewhere. Computed tomography (CT) of head revealed SAH predominantly in the right sylvian fissure, interhemispheric region, right crural cistern and the right carotid cistern (Figure 1). Digital subtraction angiography (DSA) revealed an aneurysm of the right supraclinod part of internal carotid artery (ICA) (Figure 2). The patient was referred to our institute for endovascular treatment.

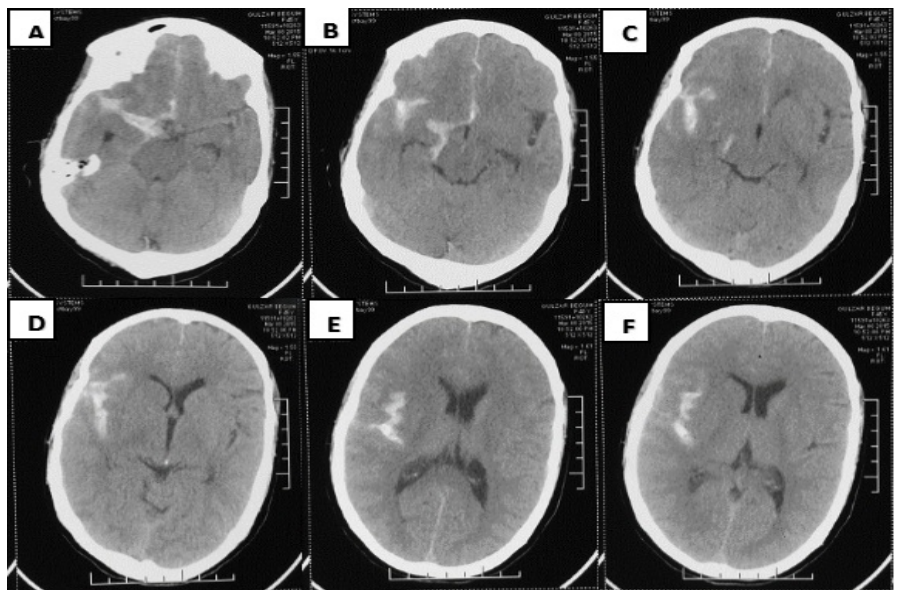

Figure 1: Subarachnoid haemorrhage predominantly on the right side after the first episode of headache and vomiting.

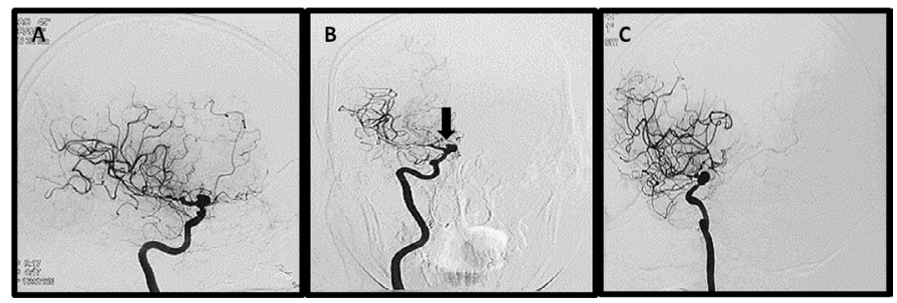

Figure 2A-B: Digital subtraction angiography done after the first episode of headache showing a saccular aneurysm in the supraclinoid part of right Internal Carotid Artery. An arrow showing a daughter sac directed towards right side (Figure - 2B)

Copy rights: (C2015 Singh, D. This is an Open access article distributed under the terms of Creative Commons Attribution 4.0 
At the time of admission, she had a Glasgow Coma Scale (GCS) of 15/15 with no neurological deficit. The time interval from the first symptom to admission at now our institute was 10 days. Patient was a known hypertensive and was on irregular treatment. There was no history of trauma. The following evening of the admission, the patient's condition deteriorated suddenly. CT scan revealed fresh subarachnoid haemorrhage (SAH). Blood was now present in the left sylvian fissure, interhemispheric region, left crural and ambient cisterns (Figure 3). The current picture of SAH was mirror image of previous SAH on opposite side. Keeping a suspicion of another aneurysm on left middle cerebral artery, the patient was taken up for endovascular coiling on emergent basis under general anaesthesia. On DSA there was no aneurysm seen on the left side but a single aneurysm was present in the supraclionid portion of right ICA which had increased in size as compared to the previous images (Figure 4). Coiling of the aneurysm was done with standard technique.

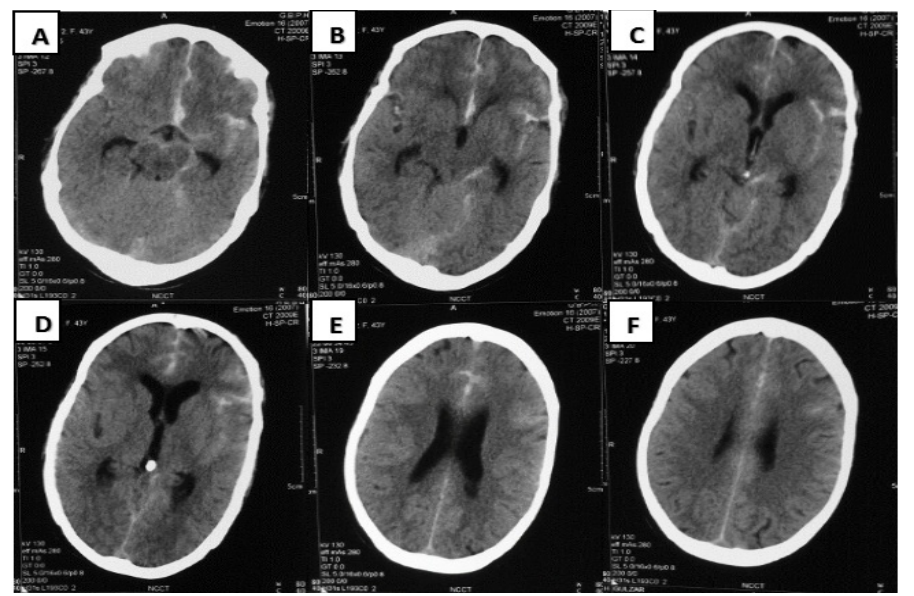

Figure 3: Subarachnoid haemorrhage predominantly on the left side after 10 days.

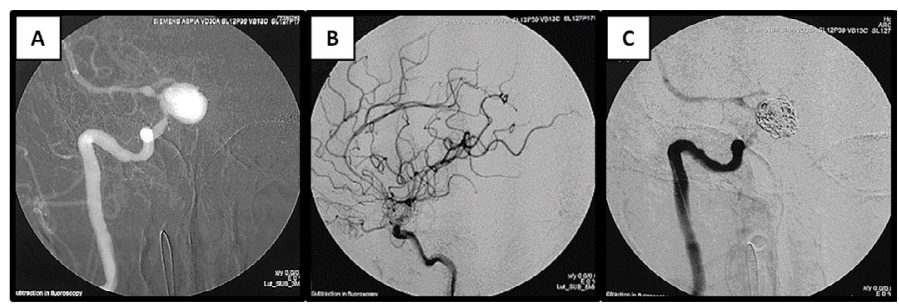

Figure 4A-C: The size of aneurysm has increased and note that the daughter sac of Figure $-2 \mathrm{~B}$ is not seen in the subsequent DSA (Figure - 4A). Coiled right supraclinoid aneurysm of right ICA (Figure $-4 \mathrm{~B}-\mathrm{C}$ ).

\section{Discussion}

Intracranial mirror aneurysms are defined as bilateral saccular aneurysms at essentially the similar locations on parent arteries in the same patient without considering the size of the aneurysms ${ }^{[3]}$. They may present with SAH on one side with incidental aneurysm on the contralateral side or rarely both may rupture on different occasions. Bilateral symmetrical aneurysms

Online ISSN: 2377-1348

Journal Title: International Journal Neurology and Brain Disorders Journal Short Name: Int J Neurol Brain Disord are usually found in the ophthalmic artery, posterior communicating artery, bifurcation of internal carotid artery, the middle cerebral artery, pericallosal artery and the posterior inferior cerebellar artery ${ }^{[4]}$. The exact incidence of mirror aneurysms is unknown. Their prevalence in the general population is unclear due to sporadic, incomplete imaging and non-standardized radiological review ${ }^{[5]}$. Variables like female sex and positive family history have been reported to be associated with mirror aneurysms but they relate more to multiplicity in general and not specifically to mirror aneurysms ${ }^{[6,7]}$. Patients with mirror aneurysms are more likely to be female and have a larger aneurysm as they have an increased frequency of aneurysm multiplicity ${ }^{[5]}$. Mirror aneurysms commonly pose a significant decision making dilemmas for intervention.

In our case the mirror picture of SAH on CT appeared as the aneurysm was directed medially, superiorly and posteriorly. It may be that the aneurysm ruptured on different sites, initially on right side and later on left. It is also possible that when the aneurysm ruptured during the first time, the blood remained on the right side cisterns and after 10 days when it re-ruptured, the blood would have traversed to the left side cisterns as adhesions may have developed in 10 days that may have prevented the blood to travel towards the right side. As the size of the aneurysm had increased as confirmed on DSA, the rupture of aneurysm on the left site cannot be entirely ruled out. A mild hydrocephalus was seen on the subsequent scan.

\section{Conclusion}

A single aneurysm of the ICA can present as mirror image SAH and can give a false suspicion of a mirror aneurysm. Mirror image SAH is a different entity than mirror aneurysm.

\section{References}

1. Baccin, C.E., Krings, T., Alvarez, H., et al. Multiple mirror-like intracranial aneurysms. Report of a case and review of the literature. (2006) ActaNeurochir(Wien) 148(10):1091-1095.

2. Rinne, J., Hernesniemi, J., Niskanen M., et al. Analysis of 561 patients with 690 middle cerebral artery aneurysms: anatomic and clinical features as correlated to management outcome. (1996) Neurosurgery $38(1): 2-11$

3. Lu, G., Huang, L., Zhang, X.L., et al. Influence of Hemodynamic Factors on Rupture of Intracranial Aneurysms: Patient-Specific 3D Mirror Aneurysms Model Computational Fluid Dynamics Simulation. (2011) Am J Neuroradiol 32(7): 1255-1261.

4. Baik, S.K., Sohn, C.H., Woo, S.K. "Mirror" Aneurysms Involving the Bilateral Distal Posterior Cerebral Artery: A Case Report of Endovascular Treatment and Literature Review. (2004) IntervNeuroradiol 10(3):231-234.

5. Meissner, I., Torner, J., Huston, J., et al. Mirror aneurysms: a reflection on natural history. (2012) J neurosurg116(6):1238-1241.

6. Rinne, J., Hernesniemi, J., Puranen, M., et al. Multiple intracranial aneurysms in a defined population: prospective angiographic and clinical study. (1994) Neurosurgery 35(5):803-808.

7. Torner, J., Wiebers, D., Brown, R., et al. Determinants of the number of intracranial aneurysms. (2005) Am J Epidemiol 161(Suppl):S27. 